\title{
Constraints of chromoelectric dipole moments to natural SUSY type sfermion spectrum
}

\author{
Nobuhiro Maekawa ${ }^{1,2 *}$, Yu Muramatsu ${ }^{3 \dagger}$ and Yoshihiro Shigekami ${ }^{1 \ddagger}$
}

\author{
${ }^{1}$ Department of Physics, Nagoya University, Nagoya 464-8602, Japan \\ ${ }^{2}$ Kobayashi Maskawa Institute, Nagoya University, Nagoya 464-8602, Japan \\ ${ }^{3}$ Institute of Particle Physics and Key Laboratory of Quark and Lepton Physics (MOE), \\ Central China Normal University, Wuhan, Hubei 430079, People's Republic of China
}

\begin{abstract}
We investigate the lower bounds of sfermion masses from the constraints of chromoelectric dipole moments (CEDMs) in the natural SUSY-type sfermion mass spectrum, in which stop mass $m_{\tilde{t}}$ is much smaller than the other sfermion masses $m_{0}$. The natural SUSY-type sfermion mass spectrum has been studied since the supersymmetric (SUSY) flavor-changing neutral currents (FCNC) are suppressed because of large sfermion masses of the first two generations, and the weak scale is stabilized because of the light stop. However, this type of sfermion mass spectrum is severely constrained by CEDM, because the light stop contributions to the up quark CEDM are not decoupled in the limit $m_{0} \rightarrow \infty$, while the down quark CEDM is decoupled in the limit. It is important that the constraints are severe even if SUSY-breaking parameters (and Higgsino mass) are taken to be real because complex diagonalizing matrices of Yukawa matrices, which are from complex Yukawa couplings, generate nonvanishing $\mathrm{CP}$ phases in off-diagonal elements of sfermion mass matrices. We calculate the CEDM of up and down quarks numerically in the minimal SUSY standard model, and give the lower bounds for stop mass and the other sfermion masses. We show that the lower bound of stop mass becomes $7 \mathrm{TeV}$ to satisfy the CEDM constraints from $\mathrm{Hg}$ EDM. The result is not acceptable if the weak scale stability is considered seriously. We show that if the up-type Yukawa couplings are taken to be real at the grand unification scale, the CEDM constraints are satisfied even if $m_{\tilde{t}} \sim 1 \mathrm{TeV}$.
\end{abstract}

*maekawa@eken.phys.nagoya-u.ac.jp

†yumura@mail.ccnu.edu.cn

${ }^{\ddagger}$ sigekami@eken.phys.nagoya-u.ac.jp 


\section{INTRODUCTION}

The supersymmetric (SUSY) extended standard model (SM) is one of the most promising candidates as the model beyond the SM. The minimal SUSY SM (MSSM) can realize the stability of the weak scale and provide a candidate of the dark matter. Moreover, it is consistent with the grand unified theory $(\mathrm{GUT})[1]$ since the three gauge couplings meet at a scale which is called the GUT scale.

However, the supersymmetry must be broken because the supersymmetric partners of the quark and lepton, which are the squark and slepton, have not been found yet, and the scale is expected to be around the weak scale, not to destabilize the weak scale. Generically, the SUSY-breaking parameters which violate the flavor and CP symmetry induce too large flavor-changing neutral current (FCNC) processes (the SUSY flavor problem) and the CP-violating processes (SUSY CP problem). To avoid these problems, we usually assume the universal sfermion masses and/or real SUSY-breaking parameters at a scale. One more interesting possibility is the decoupling solution in which the SUSY-breaking scale is taken to be much higher than the weak scale. Then the SUSY contributions to the FCNC processes and CP-violating processes are suppressed by decoupling features. The sfermion masses are required to be $O(1000) \mathrm{TeV}$ to sufficiently suppress the contribution to $\epsilon_{K}$ if off-diagonal elements of sfermion mass matrices are not suppressed[2]. This possibility has been examined more in detail since the observed Higgs mass $125 \mathrm{GeV}$ [3] requires a higher SUSY-breaking scale[4]. Unfortunately, such higher SUSY-breaking parameters result in the destabilization of the weak scale; i.e., strong fine-tuning is needed to obtain the weak scale.

One possible way to improve the fine-tuning is to make the stop mass lower, around 1 $\mathrm{TeV}$, while the other scalar fermions (sfermions) have much larger masses than $1 \mathrm{TeV}$ to suppress the FCNC and CP-violating processes. Such sfermion mass spectrum is called effective SUSY-type sfermion masses or natural SUSY[5]. Unfortunately, it has been pointed out that the sufficiently large sfermion masses are difficult to be taken since large sfermion masses and small stop mass at the GUT scale tend to result in negative stop mass square at the weak scale via two loop renormalization group effects. Large gluino mass can improve the situation, because it contributes to the stop mass square positively. Roughly, squark masses except stop mass must be smaller than 5 times gluino mass. Therefore, if the gluino mass is around $2-3 \mathrm{TeV}$, which is target of LHC experiment, $O(10) \mathrm{TeV}$ is the upper limit. Then, the off-diagonal elements of sfermion mass matrices must be suppressed. One way to suppress the off-diagonal elements is to require the modified sfermion universality in which all sfermion masses except third generation 10 dimensional fields of $S U(5)$ are universal at the GUT scale[6, 7] as

$$
\tilde{m}_{\mathbf{1 0}}^{2}=\left(\begin{array}{ccc}
m_{0}^{2} & 0 & 0 \\
0 & m_{0}^{2} & 0 \\
0 & 0 & m_{3}^{2}
\end{array}\right), \quad \tilde{m}_{\overline{\mathbf{5}}}^{2}=\left(\begin{array}{ccc}
m_{0}^{2} & 0 & 0 \\
0 & m_{0}^{2} & 0 \\
0 & 0 & m_{0}^{2}
\end{array}\right) .
$$

Such mass spectrum can be naturally obtained in $E_{6} \operatorname{GUT}[8,9,10]$ with family

symmetry[6]. Here the universality for all $\overline{\mathbf{5}}$ fields of $S U(5)$ is important to obtain 
sufficiently small FCNC processes even if the diagonalizing matrices for $\overline{\mathbf{5}}$ fields have large mixings. Therefore, when the diagonalizing matrices of $\mathbf{1 0}$ fields and $\overline{\mathbf{5}}$ fields of $S U(5)$ are estimated by Cabibbo-Kobayashi-Maskawa (CKM) matrix $V_{C K M}$ and MakiNakagawa-Sakata (MNS) matrix $V_{M N S}$ as

$$
V_{\mathbf{1 0}} \sim V_{C K M} \sim\left(\begin{array}{ccc}
1 & \lambda & \lambda^{3} \\
\lambda & 1 & \lambda^{2} \\
\lambda^{3} & \lambda^{2} & 1
\end{array}\right), \quad V_{\overline{5}} \sim V_{M N S} \sim\left(\begin{array}{ccc}
1 & \sqrt{\lambda} & \lambda \\
\sqrt{\lambda} & 1 & \sqrt{\lambda} \\
\lambda & \sqrt{\lambda} & 1
\end{array}\right)
$$

which are expected in some GUT models[10, 11], off-diagonal elements of sfermion mass matrices can be suppressed as

$$
\tilde{m}_{\mathbf{1 0}}^{2}=V_{\mathbf{1 0}}^{\dagger}\left(\begin{array}{ccc}
m_{0}^{2} & 0 & 0 \\
0 & m_{0}^{2} & 0 \\
0 & 0 & m_{3}^{2}
\end{array}\right) V_{\mathbf{1 0}}=m_{0}^{2} \mathbf{1}+\left(m_{3}^{2}-m_{0}^{2}\right)\left(\begin{array}{ccc}
\lambda^{6} & \lambda^{5} & \lambda^{3} \\
\lambda^{5} & \lambda^{4} & \lambda^{2} \\
\lambda^{3} & \lambda^{2} & 1
\end{array}\right)
$$

and most of flavor and CP constraints can be satisfied. Lepton flavor violation processes like $\mu \rightarrow e \gamma$ or $\tau \rightarrow \mu \gamma$ may be seen[12] if $m_{3} \sim O(100) \mathrm{GeV}$, but unfortunately, we lost the strong reason to take $m_{3} \sim O(100) \mathrm{GeV}$ after discovery of Higgs particle because lower bound of stop masses becomes around $1 \mathrm{TeV}$ in the MSSM in order to realize the Higgs mass $\sim 125 \mathrm{GeV}$. Here $\lambda \sim 0.22$ is the Cabibbo mixing angle.

However, even if this modified universal sfermion mass spectrum with real SUSYbreaking parameters are adopted, the EDM constraints from the experimental bound[13] as

$$
\begin{aligned}
d_{N} & <3.0 \times 10^{-26} e \mathrm{~cm} \\
d_{H g} & <7.4 \times 10^{-30} e \mathrm{~cm}
\end{aligned}
$$

become severe. The essential point is that the sfermion mass matrices of $\mathbf{1 0}$ fields of $S U(5)$ in super-CKM basis where quark and lepton mass matrices are diagonal have complex off-diagonal elements generically unless sfermion masses are universal because Yukawa couplings are complex, and therefore, diagonalizing matrices are complex to obtain the Kobayashi-Maskawa (KM) phase. Therefore, the diagram with the complex off-diagonal elements can contribute to the (chromo) EDM, and give severe constraints to the offdiagonal elements[14]. Most of contributions to (chromo) EDM are decoupled in the limit $m_{0} \rightarrow \infty$ with finite $m_{3}$, but some contributions to up quark (chromo) EDM are not decoupled in the limit[15]. The constraint becomes

$$
\operatorname{Im}\left[\frac{\left(\tilde{m}_{\mathbf{1 0}}^{2}\right)_{31}}{m_{0}^{2}} \frac{\left(\tilde{m}_{\mathbf{1 0}}^{2}\right)_{13}}{m_{0}^{2}}\right]<\left\{\begin{array}{l}
5.3 \times 10^{-6}(\mathrm{Hg}) \\
1.6 \times 10^{-4}(\text { neutron })
\end{array}\right\}\left(\frac{m_{3}}{2 \mathrm{TeV}}\right)^{2},
$$

which are obtained by the mass insertion approximation (MIA)[16] with certain mass spectrum of SUSY particles. Here we have used the relations between neutron ( $\mathrm{Hg})$ EDMs and CEDM of quarks in Ref. [17]([18]) for neutron (Hg) EDM. Although the ambiguity in theoretical calculation of EDMs is large especially for $\mathrm{Hg}[18,19]$, we give the constraints to 
the model by neglecting the uncertainty in this paper. Since $\left(\tilde{m}_{\mathbf{1 0}}^{2}\right)_{31} / m_{0}^{2} \sim\left(\tilde{m}_{\mathbf{1 0}}^{2}\right)_{13} / m_{0}^{2} \sim$ $\lambda^{3}$, the predicted EDM of Hg becomes about 20 times larger than the experimental bound even if we take stop mass $m_{\tilde{t}} \sim 2 \mathrm{TeV}$ and the diagonalizing matrices of $V_{\mathbf{1 0}}$ have small mixings like CKM matrix. This severe constraint looks to be general for almost all models with natural SUSY spectrum, and it is important to study the solution to this problem if natural SUSY spectrum is studied. Note that this problem cannot be solved by real SUSY-breaking parameters, because the CP phases of off-diagonal elements of sfermion mass matrices come from the complex Yukawa couplings which are usually assumed to obtain nonvanishing KM phase. We call this (chromo) EDM problem new SUSY CP problem in this paper.

In this paper, we focus on the new SUSY CP problem in the models with natural SUSY spectrum. One easy solution is to take large stop mass although large stop mass destabilizes the weak scale. Another one is to take diagonal up-type Yukawa matrix and therefore, diagonalizing matrix of up-type quark becomes unit matrix, although it is not so easy to obtain it in natural way. One more interesting solution to this new SUSY CP problem is to take real up-type Yukawa couplings to suppress the (chromo) EDM and complex down-type Yukawa couplings to obtain the KM phase. That possibility has been proposed and discussed in the $E_{6}$ GUT with family and CP symmetry[15, 20], which is spontaneously broken by a CP phase in Higgs vacuum expectation value (VEV) which breaks the family symmetry also.

In this paper, we calculate the chromo EDMs (CEDMs) of up and down quarks in the MSSM with different boundary conditions because the CEDMs give more severe constraints than the usual EDMs for natural SUSY-type models with real SUSY-breaking parameters. (In the recent paper[21], the EDM constraints in natural SUSY models with complex SUSY-breaking parameters has been discussed. However, the contributions discussed in this paper often give stronger constraints to the natural SUSY-type models even if SUSY-breaking parameters are real.) We also discuss the decoupling features of those constraints. First, we show that the nondecoupling feature of the up-quark CEDM contribution by the MIA and that the stop mass must be larger than $10 \mathrm{TeV}$ to satisfy the CEDM constraints. Second, we calculate the up- and down-quark CEDMs numerically in the MSSM and show that the stop mass and the other squark masses must be larger than 7 $\mathrm{TeV}$ to satisfy the up- and down-quark CEDMs. If the real up-type Yukawa couplings are adopted, CEDM constraints can be satisfied even if the stop mass is $O(1) \mathrm{TeV}$ although the other squark masses must be larger than $7 \mathrm{TeV}$. Finally, we discuss the predictions.

\section{ROUGH ESTIMATE OF CEDM}

In this section, we calculate CEDM of up quark in the modified universal sfermion mass spectrum by MIA, and see the nondecoupling feature in the limit $m_{0} \rightarrow \infty$ when $m_{3}$ and the other SUSY-breaking parameters are fixed. 
The effective lagrangian for CEDM is described as

$$
\mathcal{L}_{\mathrm{CEDM}}=-\frac{i g_{s}}{2} d_{q}^{C} \bar{q}(G \cdot \sigma) \gamma_{5} q
$$

where $g_{s}$ is the QCD coupling, $G \cdot \sigma=G_{\mu \nu}^{A} T^{A} \sigma^{\mu \nu}, G_{\mu \nu}^{A}$ is field strength of gluon, $T^{A}$ $(A=1,2, \cdots, 8)$ are $S U(3)$ generators and $\sigma^{\mu \nu}=\frac{i}{2}\left[\gamma^{\mu}, \gamma^{\nu}\right] . d_{q}^{C}$ shows quark CEDM and one can calculate this by computing diagrams shown in Fig. 1(a). In particular, $d_{q}^{C}$ is dominated by gluino contributions in the SUSY model. In the natural SUSY-type models, the diagram in Fig. 1(b) dominantly contributes to $d_{u}^{C}[14,15]$. We estimate the

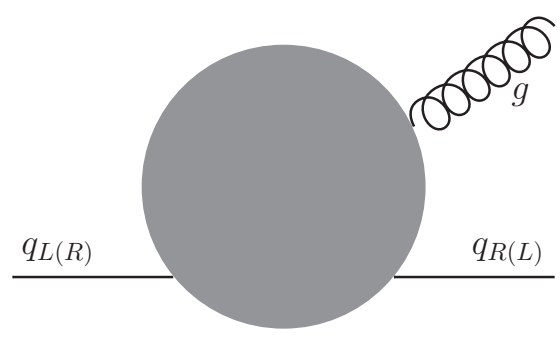

(a)

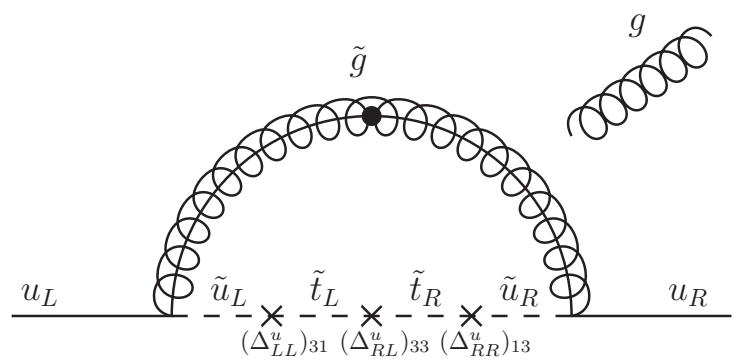

(b)

Figure 1: Diagrams contribute to $d_{q}^{C}$ (a) and $d_{u}^{C}$ (b). $\left(\Delta_{A B}^{u}\right)_{i j}(A, B=L$ or $R, i, j=$ $1,2,3)$ is the element of $6 \times 6$ sfermion mass matrix [see Eq. (10)].

magnitude of $d_{u}^{C}$ by using the diagram in Fig. 1(b) by MIA as

$$
\begin{aligned}
d_{u}^{C} & \simeq \frac{\alpha_{s}}{4 \pi} F\left(\frac{M_{\tilde{g}}^{2}}{m_{\tilde{u}}^{2}}, \frac{m_{\tilde{t}}^{2}}{m_{\tilde{u}}^{2}}\right) \operatorname{Im}\left[\frac{M_{\tilde{g}}}{m_{\tilde{t}}^{2}} \frac{\left(\Delta_{R L}^{u}\right)_{33}}{m_{\tilde{t}}^{2}} \frac{\left(\Delta_{L L}^{u}\right)_{31}}{m_{\tilde{u}}^{2}} \frac{\left(\Delta_{R R}^{u}\right)_{13}}{m_{\tilde{u}}^{2}}\right] \\
& \sim \frac{\alpha_{s}}{4 \pi} F\left(\frac{M_{\tilde{\tilde{g}}}^{2}}{m_{\tilde{u}}^{2}}, \frac{m_{\tilde{t}}^{2}}{m_{\tilde{u}}^{2}}\right) \frac{M_{\tilde{g}} A_{u 33} v_{u}}{m_{\tilde{t}}^{4}} \operatorname{Im}\left[\left(\delta_{L L}^{u}\right)_{31}\left(\delta_{R R}^{u}\right)_{13}\right]
\end{aligned}
$$

where $\left(\delta_{A B}^{u}\right)_{i j}$ are the mass insertion parameters, defined as

$$
\left(\delta_{A B}^{u}\right)_{i j} \equiv \frac{\left(\Delta_{A B}^{u}\right)_{i j}}{m_{\tilde{u}}^{2}},(A, B=L \text { or } R, i, j=1,2,3),
$$

and $F(x, y)$ is a loop function. In this definition, $\left(\Delta_{A B}^{u}\right)_{i j}$ is an element of the $6 \times 6$ sfermion mass matrix in the super-CKM basis,

$$
M_{\tilde{u}}^{2}=\left(\begin{array}{ccc}
L_{u}^{\dagger}\left(m_{Q}^{2}+v_{u}^{2} Y_{u}^{*} Y_{u}^{T}\right) L_{u} & L_{u}^{\dagger}\left(v_{u} A_{u}^{*}-\mu v_{d} Y_{u}^{*}\right) R_{u}^{*} \\
R_{u}^{T}\left(v_{u} A_{u}^{T}-\mu v_{d} Y_{u}^{T}\right) L_{u} & R_{u}^{T}\left(m_{u}^{2}+v_{u}^{2} Y_{u}^{T} Y_{u}^{*}\right) R_{u}^{*}
\end{array}\right) \equiv\left(\begin{array}{cc}
\left(\Delta_{L L}^{u}\right) & \left(\Delta_{L R}^{u}\right) \\
\left(\Delta_{R L}^{u}\right) & \left(\Delta_{R R}^{u}\right)
\end{array}\right),
$$

where $A_{u}$ is a $3 \times 3$ matrix for scalar three point vertex, $m_{Q}^{2}$ and $m_{u}^{2}$ are $3 \times 3$ soft SUSY-breaking mass matrices, and $v_{u}$ and $v_{d}$ are MSSM Higgs VEVs. Here, $L_{u}, R_{u}$ 
are diagonalizing matrices for Yukawa coupling $Y_{u}$ as $L_{u}^{T} Y_{u} R_{u}=\hat{Y}_{u}$ which is a diagonal matrix of $Y_{u}$. In the last similarity in Eq. (8), we have assumed that the gluino mass $M_{\tilde{g}}$ and $\left(\Delta_{R L}^{u}\right)_{33} \sim A_{u 33} v_{u}$ are real. Even if all SUSY-breaking parameters and Higgsino mass $\mu$ are set to be real, $M_{\tilde{u}}^{2}$ becomes complex generically because Yukawa couplings are taken to be complex to obtain the KM phase and therefore diagonalizing matrices $L_{u}$ and $R_{u}$ are complex. Quantitative constraint for mass insertion parameter in Eq. (8) comes from the current CEDM bound, $\left|d_{u}^{C}\right|<3.4 \times 10^{-27}\left(1.0 \times 10^{-25}\right) \mathrm{cm}$, which are obtained from the present upper limit of $d_{\mathrm{Hg}}\left(d_{\mathrm{N}}\right)$ in Eq. (5) [in Eq. (4)], and the relation $d_{\mathrm{Hg}} \sim 2.2 \times 10^{-3} e\left(d_{u}^{C}-d_{d}^{C}\right)[18]^{1}\left(d_{\mathrm{N}} \sim-0.3 e\left(d_{u}^{C}-d_{d}^{C}\right)[17]\right)$, as

$$
\operatorname{Im}\left[\left(\delta_{L L}^{u}\right)_{31}\left(\delta_{R R}^{u}\right)_{13}\right]<\left\{\begin{array}{l}
5.3 \times 10^{-6}(\mathrm{Hg}) \\
1.6 \times 10^{-4} \text { (neutron) }
\end{array}\right\}\left(\frac{m_{\tilde{t}}}{2 \mathrm{TeV}}\right)^{2}
$$

where we have used $g_{s} \simeq 1, m_{\tilde{t}} \sim A_{u 33} \sim 2 \mathrm{TeV}, M_{\tilde{g}} \sim 1.5 m_{\tilde{t}} \sim 3 \mathrm{TeV},{ }^{2} m_{\tilde{u}} \sim 10 \mathrm{TeV}$, and the loop integral function $F(0.09,0.04)$ is 0.079 which is obtained from the Appendix. When we assume that

$$
L_{u}, R_{u} \sim V_{\mathrm{CKM}} \sim\left(\begin{array}{ccc}
1 & \lambda & \lambda^{3} \\
\lambda & 1 & \lambda^{2} \\
\lambda^{3} & \lambda^{2} & 1
\end{array}\right)
$$

and $m_{\tilde{u}}=m_{\tilde{c}} \gg m_{\tilde{t}}$, the left-hand side of Eq. (11) becomes

$$
\operatorname{Im}\left[\left(\delta_{L L}^{u}\right)_{31}\left(\delta_{R R}^{u}\right)_{13}\right] \simeq\left(\frac{m_{\tilde{t}}^{2}-m_{\tilde{u}}^{2}}{m_{\tilde{u}}^{2}}\right)^{2} \times \lambda^{6}(\lambda=0.22) .
$$

So this contribution does not decouple in the limit of $m_{\tilde{u}} \rightarrow \infty$ and the size is about $10^{-4}$ that is about 20 times larger than the constraint from $\mathrm{Hg}$ EDM. Therefore, $m_{\tilde{t}}>10 \mathrm{TeV}$ is required to satisfy the CEDM constraint in this approximation.

On the other hand, for the CEDM of down quark, $d_{d}^{C}$, such contributions from flavorviolating mass insertion is decoupled when sdown mass $m_{\tilde{d}} \rightarrow \infty$ because the right-handed sbottom mass $m_{\tilde{b}} \sim m_{\tilde{d}}$. Therefore, $m_{\tilde{d}}>10 \mathrm{TeV}$ is expected to be required to satisfy the experimental bound of CEDM if the decoupling feature is similar to that of $d_{u}^{C}$ for $m_{\tilde{t}}$.

Therefore, the CEDM of up quark is more serious in natural SUSY scenario. One simple solution is that real $Y_{u}$ and $A_{u}$ are taken while $Y_{d}$ is complex that induces the KM phase. In this case, diagonalizing matrices of up-type quark mass matrix are also real and then $d_{u}^{C}$ is strongly suppressed. Note that $Y_{u}$ becomes complex through the renormalization group equation (RGE), even if $Y_{u}$ is real at the GUT scale. In such a case, however, $d_{u}^{C}$ is small enough to satisfy the current experimental bound as we will show in Sec. 3.

\footnotetext{
${ }^{1}$ Here, we use the bound for $\left|d_{u}^{C}-d_{d}^{C}\right|$ as the bound for $d_{u}^{C}$. This is justified in the limit $m_{0} \rightarrow \infty$ because $d_{d}^{C}$ is vanishing in the limit. In this paper, we just use the bound for $d_{u}^{C}-d_{d}^{C}$ as the bound for $d_{u}^{C}$ and $d_{d}^{C}$ even with finite $m_{0}$.

${ }^{2}$ If $A$-term is smaller, the constraints become weaker, although $A \ll M_{\tilde{g}}$ usually requires a tuning in the MSSM with SUSY-breaking parameters given at the GUT scale because of the renormalization group effects.
} 


\section{EVALUATIONS AND RESULTS}

In this section, we numerically calculate the CEDM in the MSSM with the modified universal sfermion mass spectrum.

Now, we explain the procedure for the calculation of CEDMs. First of all, input parameters, which are gauge couplings $g_{i}$, gaugino masses $M_{i}$, Yukawa couplings, $A$ parameters which are couplings of three scalar's vertex, sfermion mass matrices, and doublet Higgs masses are given at the GUT scale, $\Lambda_{\mathrm{G}}=2 \times 10^{16} \mathrm{GeV}$, as

$$
\begin{aligned}
& g_{1}\left(\Lambda_{\mathrm{G}}\right)=g_{2}\left(\Lambda_{\mathrm{G}}\right)=g_{3}\left(\Lambda_{\mathrm{G}}\right)=g_{\mathrm{GUT}}=0.7, \\
& M_{1}\left(\Lambda_{\mathrm{G}}\right)=M_{2}\left(\Lambda_{\mathrm{G}}\right)=M_{3}\left(\Lambda_{\mathrm{G}}\right)=M_{1 / 2}, \\
& Y_{u} \sim\left(\begin{array}{ccc}
\lambda^{6} & \lambda^{5} & \lambda^{3} \\
\lambda^{5} & \lambda^{4} & \lambda^{2} \\
\lambda^{3} & \lambda^{2} & 1
\end{array}\right), \quad A_{u} \sim A_{0}\left(\begin{array}{ccc}
\lambda^{6} & \lambda^{5} & \lambda^{3} \\
\lambda^{5} & \lambda^{4} & \lambda^{2} \\
\lambda^{3} & \lambda^{2} & 1
\end{array}\right), \\
& Y_{d} \sim Y_{e}^{T} \sim\left(\begin{array}{ccc}
\lambda^{6} & \lambda^{5.5} & \lambda^{5} \\
\lambda^{5} & \lambda^{4.5} & \lambda^{4} \\
\lambda^{3} & \lambda^{2.5} & \lambda^{2}
\end{array}\right), \quad A_{d} \sim A_{e}^{T} \sim A_{0}\left(\begin{array}{ccc}
\lambda^{6} & \lambda^{5.5} & \lambda^{5} \\
\lambda^{5} & \lambda^{4.5} & \lambda^{4} \\
\lambda^{3} & \lambda^{2.5} & \lambda^{2}
\end{array}\right), \\
& \tilde{m}_{\mathbf{1 0}}^{2}=\left(\begin{array}{ccc}
m_{0}^{2} & 0 & 0 \\
0 & m_{0}^{2} & 0 \\
0 & 0 & m_{3}^{2}
\end{array}\right), \quad \tilde{m}_{\overline{\mathbf{5}}}^{2}=\left(\begin{array}{ccc}
m_{0}^{2} & 0 & 0 \\
0 & m_{0}^{2} & 0 \\
0 & 0 & m_{0}^{2}
\end{array}\right), \\
& m_{H_{u}}^{2}\left(\Lambda_{\mathrm{G}}\right)=m_{H_{d}}^{2}\left(\Lambda_{\mathrm{G}}\right)=(500 \mathrm{GeV})^{2},
\end{aligned}
$$

where $A_{0}$ is the typical scale of $A$ parameters, and sfermion mass matrices $\tilde{m}_{10}^{2}$ and $\tilde{m}_{\overline{\mathbf{5}}}^{2}$ are for $\mathbf{1 0}$ and $\overline{\mathbf{5}}$ fields, respectively. The Higgsino mass $\mu$ is fixed by the value of the $\mathrm{Z}$ boson mass $m_{Z}$. ( $\mu$ becomes comparatively large $(O(1) \mathrm{TeV})$, which may destabilize the weak scale. But we do not mind it because large $\mu$ does not contribute much to $d_{u}^{C}$.) Next, in order to obtain low-energy parameters from these inputs, we use two-loop RGEs based on Ref. [22]. Note that, for simplicity, we consider MSSM from GUT scale to the SUSY-breaking scale. Finally, we compute up, down and strange quark CEDMs. These CEDMs denoted as $d_{q}^{C}(q=u, d, s)$ are evaluated by the following one-loop formulas,

$$
\begin{aligned}
& d_{u}^{C}=c \frac{\alpha_{s}}{4 \pi} \sum_{j=1}^{6} \frac{M_{\tilde{g}}}{\left(\hat{M}_{\tilde{u}}^{2}\right)_{j j}}\left\{\left(-\frac{1}{3} F_{1}\left(x_{j}^{u}\right)-3 F_{2}\left(x_{j}^{u}\right)\right) \operatorname{Im}\left[\left(U_{\tilde{u}}^{\dagger}\right)_{1 j}\left(U_{\tilde{u}}\right)_{j 4}\right]\right\}, \\
& d_{d}^{C}=c \frac{\alpha_{s}}{4 \pi} \sum_{j=1}^{6} \frac{M_{\tilde{g}}}{\left(\hat{M}_{\tilde{d}}^{2}\right)_{j j}}\left\{\left(-\frac{1}{3} F_{1}\left(x_{j}^{d}\right)-3 F_{2}\left(x_{j}^{d}\right)\right) \operatorname{Im}\left[\left(U_{\tilde{d}}^{\dagger}\right)_{1 j}\left(U_{\tilde{d}}\right)_{j 4}\right]\right\}, \\
& d_{s}^{C}=c \frac{\alpha_{s}}{4 \pi} \sum_{j=1}^{6} \frac{M_{\tilde{g}}}{\left(\hat{M}_{\tilde{d}}^{2}\right)_{j j}}\left\{\left(-\frac{1}{3} F_{1}\left(x_{j}^{d}\right)-3 F_{2}\left(x_{j}^{d}\right)\right) \operatorname{Im}\left[\left(U_{\tilde{d}}^{\dagger}\right)_{2 j}\left(U_{\tilde{d}}\right)_{j 5}\right]\right\},
\end{aligned}
$$

where $c \sim 0.9$ is QCD correction. $\hat{M}_{\tilde{q}}^{2}(q=u, d)$ are diagonalized squark mass matrices which are obtained as $\hat{M}_{\tilde{q}}^{2}=U_{\tilde{q}} M_{\tilde{q}}^{2} U_{\tilde{q}}^{\dagger}$, where $M_{\tilde{q}}^{2}$ and $U_{\tilde{q}}$ are $6 \times 6$ squark mass matrices 
and the diagonalizing matrices, respectively. $F_{1}(x)=\left(x^{2}-4 x+3+2 \ln x\right) / 2(1-x)^{3}$ and $F_{2}(x)=\left(x^{2}-1-2 x \ln x\right) / 2(1-x)^{3}$ are coming from loop integrals and $x_{j}^{q}=\frac{M_{\tilde{g}}^{2}}{\left(\hat{M}_{\tilde{q}}^{2}\right)_{j j}}$. The current bounds $[13,14]$ are

$$
\begin{aligned}
& \left|d_{q}^{C}\right|<3.4 \times 10^{-27} \mathrm{~cm} \quad(q=u, d),(\mathrm{Hg}) \\
& \left|d_{q}^{C}\right|<1.0 \times 10^{-25} \mathrm{~cm} \quad(q=u, d),(\text { neutron }) \\
& \left|d_{s}^{C}\right|<1.1 \times 10^{-25} \mathrm{~cm} .
\end{aligned}
$$

We consider three types of inputs of Yukawa couplings and $A$ parameters. All of three types have the hierarchy explained above, but have different type of $\mathcal{O}(1)$ coefficients. To explain this, we show the explicit forms of these matrices:

$$
\begin{aligned}
& Y_{u}=\left(\begin{array}{ccc}
y_{u 11} \lambda^{6} & y_{u 12} \lambda^{5} & y_{u 13} \lambda^{3} \\
y_{u 21} \lambda^{5} & y_{u 22} \lambda^{4} & y_{u 23} \lambda^{2} \\
y_{u 31} \lambda^{3} & y_{u 32} \lambda^{2} & y_{u 33}
\end{array}\right), \quad A_{u}=A_{0}\left(\begin{array}{ccc}
a_{u 11} \lambda^{6} & a_{u 12} \lambda^{5} & a_{u 13} \lambda^{3} \\
a_{u 21} \lambda^{5} & a_{u 22} \lambda^{4} & a_{u 23} \lambda^{2} \\
a_{u 31} \lambda^{3} & a_{u 32} \lambda^{2} & 1
\end{array}\right) \\
& Y_{d}=\left(\begin{array}{lll}
y_{d 11} \lambda^{6} & y_{d 12} \lambda^{5.5} & y_{d 13} \lambda^{5} \\
y_{d 21} \lambda^{5} & y_{d 22} \lambda^{4.5} & y_{d 23} \lambda^{4} \\
y_{d 31} \lambda^{3} & y_{d 32} \lambda^{2.5} & y_{d 33} \lambda^{2}
\end{array}\right), \quad A_{d}=A_{0}\left(\begin{array}{lll}
a_{d 11} \lambda^{6} & a_{d 12} \lambda^{5.5} & a_{d 13} \lambda^{5} \\
a_{d 21} \lambda^{5} & a_{d 22} \lambda^{4.5} & a_{d 23} \lambda^{4} \\
a_{d 31} \lambda^{3} & a_{d 32} \lambda^{2.5} & a_{d 33} \lambda^{2}
\end{array}\right) \\
& Y_{e}=\left(\begin{array}{ccc}
y_{e 11} \lambda^{6} & y_{e 12} \lambda^{5} & y_{e 13} \lambda^{3} \\
y_{e 21} \lambda^{5.5} & y_{e 22} \lambda^{4.5} & y_{e 23} \lambda^{2.5} \\
y_{e 31} \lambda^{5} & y_{e 32} \lambda^{4} & y_{e 33} \lambda^{2}
\end{array}\right), \quad A_{e}=A_{0}\left(\begin{array}{ccc}
a_{e 11} \lambda^{6} & a_{e 12} \lambda^{5} & a_{e 13} \lambda^{3} \\
a_{e 21} \lambda^{5.5} & a_{e 22} \lambda^{4.5} & a_{e 23} \lambda^{2.5} \\
a_{e 31} \lambda^{5} & a_{e 32} \lambda^{4} & a_{e 33} \lambda^{2}
\end{array}\right)
\end{aligned}
$$

- real $Y_{u}$ type

At the GUT scale, $y_{d i j}, a_{d i j}, y_{e i j}$ and $a_{e i j}$ are complex $\mathcal{O}(1)$ coefficients, while $y_{u i j}$ and $a_{u i j}$ are real $\mathcal{O}(1)$ coefficients $(i, j=1,2,3)$.

- complex $Y_{u}$ type

All $y_{u i j}, a_{u i j}, y_{d i j}, a_{d i j}, y_{e i j}$ and $a_{e i j}$ are complex $\mathcal{O}(1)$ coefficients $(i, j=1,2,3)$.

- $E_{6}$ model (with family symmetry and spontaneous CP violation)

There are special relations obtained in the model in Ref.[20]: $y_{u 11}=y_{u 13}=y_{u 31}=$ $y_{e 13}=y_{e 21}=0, y_{u 12}=-y_{u 21}=y_{d 13}=\frac{1}{3} d_{q}, y_{u 23}=y_{u 32}, y_{d 23}=y_{e 32}, y_{d 33}=y_{e 33}$ and $y_{e 12}=-y_{e 31} \cdot y_{d 11}, y_{d 12}, y_{d 22}, y_{d 32}, y_{e 11}, y_{e 22}$ and $y_{e 23}$ are complex $\mathcal{O}(1)$ coefficients, and $d_{q}, y_{u 22}, y_{u 23}, y_{u 33}, y_{d 21}, y_{d 23}, y_{d 31}, y_{d 33}$ and $y_{e 12}$ are real $\mathcal{O}(1)$ coefficients (there are same structures in $A$ parameters).

For all types, we take real parameters for $M_{1 / 2}, \mu, A_{u 33}=A_{0}$, and $y_{u 33}=0.8$ at the GUT scale and we set $\tan \beta=7$. In these parameters, most of the usual contributions to EDMs are strongly suppressed especially when $m_{0} \rightarrow \infty$. We take $A$ parameters which have similar hierarchies to the corresponding Yukawa couplings. This situation can be realized in models in which hierarchies of Yukawa couplings are explained by the FroggattNielsen mechanism[23]. In such situation, we think it reasonable that $O(1)$ coefficients of 
$A$ parameters are complex number when the $O(1)$ coefficients of corresponding Yukawa couplings are complex as in Ref. $[6,15,20]$. We checked that the numerical results do not change at all if all $O(1)$ coefficients of $A$ parameters are taken to be real for all types above.

We need to make a few comments on $\mathcal{O}(1)$ coefficient. The complex $\mathcal{O}(1)$ coefficient $C$ means that $C=|C| \exp \left(i \theta^{(C)}\right)$ as $|C|$ is real $\mathcal{O}(1)$ coefficient and $\theta^{(C)}$ is random number in the ranges $0 \leq \theta^{(C)} \leq 2 \pi$. In addition, real $\mathcal{O}(1)$ coefficient means random number within the interval 0.5 to 1.5 with + or - signs $^{3}$.

We have calculated CEDMs in $O(100)$ model points with different $O(1)$ coefficients and obtained the average and the standard deviation of $\log _{10}\left|d_{q}^{C}\right|$ which are shown in Figs. 2-5 as the center value and the error bar, respectively. For simplicity, we do not impose the conditions to obtain realistic CKM matrix in our calculation. The result is below. First we show $m_{0}\left(m_{\tilde{d}}\right)$ dependence of CEDMs in Fig. 2. The vertical axis is $\log _{10}\left|d_{u}^{C}\right|$ (upper panel) and $\log _{10}\left|d_{d}^{C}\right|$ (lower panel), and the horizontal axis is heavy sfermion mass at low energy denoted as $m_{\tilde{d}}$, which is almost determined by $m_{0}$ value. Red, blue and green plots are real $Y_{u}$ type, complex $Y_{u}$ type and $E_{6}$ model, respectively. Black solid line is current bound from $\mathrm{Hg} \mathrm{EDM}$ and allowed region is lower area. Dashed line shows the current bound from neutron EDM, and the dotted line is the bound expected in future experiments of neutron $\operatorname{EDM}[24]$. We set $A_{0}=-1 \mathrm{TeV}$ at the GUT scale. In these figures, we choose $M_{1 / 2}$ and $m_{3}$ so that stop mass at the SUSY scale ${ }^{4}$ become about 2 $\mathrm{TeV}$ in each $m_{0}$ case, and $M_{\tilde{g}}$ and $\left|A_{u 33}\right|$ value at the SUSY scale is shown in Table I. From Fig. 2, it is clear that $d_{d}^{C}$ is decoupled when $m_{0}$ increases and we found that roughly $m_{\tilde{d}}>7 \mathrm{TeV}$ is required to satisfy the current bound from Hg EDM, corresponding to the situation setting $m_{0}>7 \mathrm{TeV}$ at GUT scale. (In this paper, we discuss the limit of sfermion masses by using the center value in the distribution.) However, because of nondecoupling effect caused by stop contribution, current bound for $d_{u}^{C}$ is severe if $Y_{u}$ is complex at GUT scale. In order to satisfy the current bound in the complex $Y_{u}$ type, $m_{\tilde{t}}$ must be larger.

How large $m_{\tilde{t}}$ is required to suppress $d_{u}^{C}$ sufficiently? We show $m_{\tilde{t}}$ dependence of CEDMs in Fig. 3. The vertical axis is $\log _{10}\left|d_{u}^{C}\right|$ (upper panel) and $\log _{10}\left|d_{d}^{C}\right|$ (lower panel), and the horizontal axis is $m_{\tilde{t}}$. The colors of plots and shapes of lines have the same meanings as in Fig. 2. In these figures, we set $m_{0}=20 \mathrm{TeV}$ and $A_{0}=-1 \mathrm{TeV}$ at GUT scale. $M_{\tilde{g}}=3 \mathrm{TeV}$ and $m_{\tilde{t}} \equiv \sqrt{m_{\tilde{t} 1} m_{\tilde{t} 2}}$ are given at the SUSY scale, where $m_{\tilde{t}_{1}}^{2}$ and $m_{\tilde{t}_{2}}^{2}$ are eigenvalues of the matrix of stop mass square. Then $A_{u 33} \sim 2.2 \mathrm{TeV}$ at the SUSY scale. From Fig. 3, it is easy to understand that $d_{u}^{C}$ is strongly dependent on $m_{\tilde{t}}$, while $d_{d}^{C}$ is almost independent. The flat regions appear also in $d_{u}^{C}$, which are caused by the contributions from the first two generation squarks. Roughly, when $m_{\tilde{t}}>7 \mathrm{TeV}$, current bound of $d_{u}^{C}$ from $\mathrm{Hg}$ EDM is satisfied even if $Y_{u}$ is complex at GUT scale. (Note that

\footnotetext{
${ }^{3}$ We don't contain one-loop threshold corrections in the calculation because $\mathcal{O}(1)$ coefficients produce a much greater difference in the results of CEDM than the threshold correction, although we should take into account the one-loop threshold corrections when we consider two-loop RGEs.

${ }^{4}$ In this paper, we take the SUSY scale $=1 \mathrm{TeV}$ as the renormalization scale, even when the squark masses are much larger than $1 \mathrm{TeV}$.
} 


\begin{tabular}{|c||c|c|}
\hline$m_{0}(\mathrm{TeV})$ & $M_{\tilde{g}}(\mathrm{TeV})$ & $\left|A_{u 33}\right|(\mathrm{TeV})$ \\
\hline \hline 5 & 2.7 & 2.0 \\
\hline 10 & 2.8 & 2.1 \\
\hline 20 & 4.3 & 3.1 \\
\hline 30 & 6.2 & 4.4 \\
\hline 40 & 8.4 & 5.8 \\
\hline
\end{tabular}

Table I: $M_{\tilde{g}}$ and $\left|A_{u 33}\right|$ at the SUSY scale $(1 \mathrm{TeV})$ in each $m_{0}$ value for calculation in Fig. 2 .

this lower limit for $m_{\tilde{t}}$ is not so far from the prediction obtained by the MIA as well as the lower limit for $m_{\tilde{d}}$. ) However, from the point of view of naturalness, it is preferable that $m_{\tilde{t}}$ has smaller value, so such a large stop mass may not be acceptable. real $Y_{u}$ type and $E_{6}$ model are satisfying $d_{u}^{C}$ bound even if the stop mass is smaller than $1 \mathrm{TeV}$. Therefore, real $Y_{u}$ at GUT scale can be an important condition to satisfy $d_{u}^{C}$ bound when $m_{\tilde{t}} \sim \mathcal{O}(1)$ $\mathrm{TeV}$.

We have investigated $m_{\tilde{d}}$ or $m_{\tilde{t}}$ dependence of strange quark CEDM $d_{s}^{C}$. The results are very similar to $d_{d}^{C}$ results and constraints of $d_{s}^{C}$ are much weaker than that of $d_{d}^{C}$ (see Fig. 4.), and therefore, we do not discuss the strange quark CEDM in detail in this paper.

Finally, we check whether $125 \mathrm{GeV}$ Higgs mass is really obtained in our setup. To do this, we use FeynHiggs-2.10 [25]. We set GUT scale parameters as shown in Table II. $M_{1 / 2}, m_{3}$, and $A_{0}$ are chosen so that all sfermions have positive squared masses at SUSY scale. We also show $m_{\tilde{t}}$ and $\left|A_{u 33}\right|$ at SUSY scale in Table III. We found that the $125 \mathrm{GeV}$ Higgs mass is realized in all three types. The values of CEDMs in each situation are

\begin{tabular}{|c||c|c|c|}
\hline$m_{0}(\mathrm{TeV})$ & $m_{3}(\mathrm{TeV})$ & $M_{1 / 2}(\mathrm{TeV})$ & $A_{0}(\mathrm{TeV})$ \\
\hline \hline 5 & 1.2 & 1.5 & -5.0 \\
\hline 10 & 1.5 & 1.8 & -4.5 \\
\hline 20 & 2.0 & 2.4 & -2.5 \\
\hline 40 & 3.5 & 4.5 & -3.5 \\
\hline
\end{tabular}

Table II: GUT scale parameters which we use in each $m_{0}$ value.

shown in Fig. 4. Upper panel is $d_{u}^{C}$ versus $d_{d}^{C}$, and lower panel is $d_{u}^{C}$ versus $d_{s}^{C}$. In these figures, diamond, square and circle plots are corresponding to real $Y_{u}$ type, complex $Y_{u}$ type and $E_{6}$ model, respectively. Red, blue, green and orange means that $m_{0}$ is $5 \mathrm{TeV}, 10$ $\mathrm{TeV}, 20 \mathrm{TeV}$ and $40 \mathrm{TeV}$. Each error bar shows the standard deviation for the predicted values of $\log _{10}\left|d_{q}^{C}\right|(q=u, d, s)$ which are obtained in various model points with different $O(1)$ coefficients. Black solid line is current bound from $\mathrm{Hg}$ EDM and allowed region is lower left area. Dashed line shows the current bound from neutron EDM, and the dotted line is the bound expected in future experiments of neutron EDM. From Fig. 4, it is clear that $d_{u}^{C}$ bound for complex $Y_{u}$ type is severe, even when $125 \mathrm{GeV}$ Higgs mass is realized. 


\begin{tabular}{|c||c|c|}
\hline$m_{0}(\mathrm{TeV})$ & $m_{\tilde{t}}(\mathrm{TeV})$ & $\left|A_{u 33}\right|(\mathrm{TeV})$ \\
\hline \hline 5 & 1.9 & 3.4 \\
\hline 10 & 2.3 & 3.7 \\
\hline 20 & 2.6 & 4.1 \\
\hline 40 & 4.3 & 7.4 \\
\hline
\end{tabular}

Table III: $m_{\tilde{t}}$ and $\left|A_{u 33}\right|$ at SUSY scale in each $m_{0}$ value.

In $E_{6}$ model, each CEDM value is smaller than that of the other two types because of the special structures of $Y_{u}$ and $A_{u}$ at the GUT scale. Therefore, these structures have some effects to suppress $\left|d_{u}^{C}\right|$ value.

\section{COMMENT ON ELECTRON EDM}

Recently, the constraint of electron EDM, $d_{e}$, is improved [26] and may be severe for this discussion. Then we also check the constraint of $d_{e}$ in the same situations discussed above. Note that we evaluate $d_{e}$ by using the expression based on Ref. [27]. Although there are mainly two types of contributions to $d_{e}$ in SUSY model, neutralino and chargino contributions, we ignore the chargino contributions because Higgsinos are heavier than wino. So, we will show the $d_{e}$ result for the sum of four neutralino contributions in our setup.

The result is shown in Fig. 5. The vertical axis is $\log _{10}\left|d_{e}\right|$. In this case, we set horizontal axis as slepton mass at low energy, which is almost determined by $m_{0}$ value. Red, blue and green plots are the real $Y_{u}$ type, complex $Y_{u}$ type and $E_{6}$ model, respectively. Black solid line is current bound, $\left|d_{e}\right|<8.7 \times 10^{-29} e \mathrm{~cm}[26]$, and allowed region is lower area. Other input parameters which are used for calculation are the same as for the Fig. 2. Neutralino masses at SUSY scale in each $m_{0}$ case are shown in Table IV.

\begin{tabular}{|c||c|c|c|}
\hline$m_{0}(\mathrm{TeV})$ & $m_{N_{1}}(\mathrm{TeV})$ & $m_{N_{2}}(\mathrm{TeV})$ & $m_{N_{3}}, m_{N_{4}}(\mathrm{TeV})$ \\
\hline \hline 5 & 0.5 & 0.9 & 2.1 \\
\hline 10 & 0.6 & 1.0 & 2.4 \\
\hline 20 & 0.8 & 1.5 & 3.3 \\
\hline 30 & 1.2 & 2.2 & 4.2 \\
\hline 40 & 1.6 & 3.0 & 5.2 \\
\hline
\end{tabular}

Table IV: Neutralino masses at SUSY scale in each $m_{0}$ case. In this calculation, the masses of two heavy neutralinos, $m_{N_{3}}$ and $m_{N_{4}}$, are almost degenerated.

Compared with Figs. 2 and 5, we found that constraint of $d_{e}$ is weaker than that of $d_{d}^{C}$ at least in the situation we discussed in this paper. Roughly speaking, $m_{\tilde{e}}>6$ or $7 \mathrm{TeV}$ is required for the $d_{e}$ bound in real $Y_{u}$ type and complex $Y_{u}$ type while $d_{e}$ bound is still 
satisfied with $m_{\tilde{e}}>5 \mathrm{TeV}$ in the $E_{6}$ model. This is because there are special structures not only for $Y_{u}$ and $Y_{d}$ but also for the $Y_{e}$ in the $E_{6}$ model. Electron EDM experiments will be improved in a few years [28], so we should care about not only the CEDM bounds but also this bound. The expected future bounds are presented in Fig. 5. Interestingly, we can expect a signal of the electron EDM in the future. If it is not seen, $m_{0}$ must be larger than $40 \mathrm{TeV}$.

\section{SUMMARY AND DISCUSSION}

In this paper, we discussed the CEDM bounds in the SUSY model with the natural SUSY-type sfermion mass spectrum in which the stop masses $m_{\tilde{t}}$ are $O(1) \mathrm{TeV}$ while the other squark masses $m_{0}$ are much larger than $m_{\tilde{t}}$ since the CEDM constraints, especially from the Hg EDM, give severe constraint to this natural SUSY-type sfermion mass spectrum even if real SUSY-breaking parameters are assumed. We calculated the CEDM of up, down, and strange quarks numerically at the three boundary conditions for Yukawa couplings at the GUT scale, the real $Y_{u}$ type, complex $Y_{u}$ type, and the $E_{6}$ model, and discussed their decoupling features. First, we concluded that the up-quark CEDM becomes sufficiently small to satisfy the experimental bound when up-quark Yukawa couplings are real at the GUT scale even if we take $m_{\tilde{t}} \sim O(1) \mathrm{TeV}$ not to destabilize the weak scale, while it becomes too large when the Yukawa couplings are complex even if $m_{0} \gg m_{\tilde{t}}$. On the other hand, the down and strange quark CEDM become sufficiently small if $m_{0}>7$ $\mathrm{TeV}$ because of the decoupling feature. Second, to satisfy the up-quark CEDM constraint with complex $Y_{u}, m_{\tilde{t}}>7 \mathrm{TeV}$ is needed, which destabilizes the weak scale.

In the natural SUSY-type sfermion mass spectrum, off-diagonal elements of $\left(\Delta_{R R}^{d}\right)$, which is defined as the same rule in Eq. (10) are strongly suppressed because the masses of the right-handed sdown type are almost degenerated. For this reason, a dominant contribution to $d_{d}^{C}$ is proportional to $\operatorname{Im}\left[\left(\Delta_{L L}^{d}\right)_{31}\left(\Delta_{R L}^{d}\right)_{13}\right]\left(\propto A_{d 13}\right.$ in the super-CKM basis $)$ rather than $\operatorname{Im}\left[\left(\Delta_{L L}^{d}\right)_{31}\left(\Delta_{R L}^{d}\right)_{33}\left(\Delta_{R R}^{d}\right)_{13}\right]$. When the $A$ parameters are proportional to the corresponding Yukawa couplings $A_{f} \propto Y_{f}(f=u, d, e)$ at the GUT scale, off-diagonal elements of $A$ parameters in the super-CKM basis are suppressed at the SUSY-breaking scale and, therefore, $d_{d}^{C}$ is strongly suppressed $\left(\sim \mathcal{O}\left(10^{-32}\right) e \mathrm{~cm}\right)$. In such a case, we cannot constrain the sfermion masses from the bound of $d_{d}^{C}$ even when the CEDM constraints are improved at the level of the future neutron EDM sensitivity. Note that this situation can be also realized when $A_{0}=0$ at the GUT scale. On the other hand, in the case of $A_{f} \propto Y_{f}$ at the GUT scale, $d_{u}^{C}$ does not change so much in the natural SUSY-type sfermion mass spectrum because the dominant contribution to $d_{u}^{C}$ is proportional to the diagonal element of the $A$ parameter $A_{u 33}$ in the super-CKM basis as discussed in Eq. (8). The situation does not change so much in the case of $A_{0}=0$ at the GUT scale since the $A_{0}$ value does not affect the value of the diagonal elements of the $A$ parameters at the SUSY-breaking scale so much because of the RGE running. We checked these behaviors numerically, and the lower bounds of $m_{\tilde{t}}$ from the bound of $d_{u}^{C}$ are the same as our results.

These constraints are dependent on explicit models for Yukawa couplings and the 
sfermion mass spectrum. In this paper, we have just demonstrated the constraints from the EDMs in a certain model for the Yukawa couplings and the sfermion mass spectrum, which are obtained from the $E_{6}$ GUT with family symmetry. Therefore, the constraints become different from ours if different models for Yukawa couplings are adopted. However, we note that our model will give comparatively weaker constraints than the others because the diagonalizing matrices of the up-type Yukawa matrix have small mixings. (Of course, we can consider the models which give weaker constraints than ours, for example, the diagonal up-type Yukawa matrix model.) Therefore, our constraints to the natural SUSYtype sfermion mass models from $d_{u}^{C}$ are quite general.

In this paper, we have neglected the uncertainties in the calculation of the relation between the $\mathrm{Hg}$ (neutron) EDM and CEDMs and discussed the constraints. However, the uncertainties for the coefficients are more than $100 \%$ for the $\mathrm{Hg}$ EDM. Conservatively, we may have to use the constraints only from the neutron EDM. Then, we have almost no constraints from the neutron EDM to the natural SUSY model with $O(1)$ TeV stop mass. In that case, constraints from the electron EDM become important and give lower bounds of $m_{0}$, although no constraint for $m_{\tilde{t}}$ is given. Since the experimental sensitivity of the electron EDM is expected to be improved by about 2 orders of magnitude, we can expect that nonvanishing electron EDM is observed in future experiments. If it is not observed, the $m_{0}$ is expected to be larger than $40 \mathrm{TeV}$. To improve the bound for $m_{\tilde{t}}$, future experiments of neutron EDM are important. Since experimental sensitivity of the neutron EDM is expected to be improved by more than two orders of magnitude, the observation of nonvanishing EDM of neutron is expected. No signal means $m_{0}>20$ $\mathrm{TeV}$, and $m_{\tilde{t}}>20 \mathrm{TeV}$ if $Y_{u}$ is complex, while almost no constraint to $m_{\tilde{t}}$ if $Y_{u}$ is real. One more way to improve the bound for $m_{\tilde{t}}$ is, of course, to reduce the uncertainties in theoretical calculation of $\mathrm{Hg}$ EDM.

We conclude that the up quark CEDM constraint can be severe in natural SUSY type sfermion mass spectrum. If experimental bounds of EDM and/or theoretical calculation of $\mathrm{Hg} \mathrm{EDM}$ are improved in future, they will constrain the lower bounds of stop mass and the other heavy sfermion masses. In addition, if the sfermion masses, especially the stop mass, are observed in future experiments, we may be able to constrain the structure of $Y_{u}$ at GUT scale by the CEDM constraints.

\section{ACKNOWLEDGMENTS}

We thank F. Sala and J. Hisano for advice on the present situation of theoretical calculation of Hg EDM. N. M. is supported in part by Grants-in-Aid for Scientific Research from MEXT of Japan (No. 15K05048). Y.M. is supported in part by the National Research Foundation of Korea (NRF) Research Grant No. NRF- 2015R1A2A1A05001869 and the National Natural Science Foundation of China (NSFC) under Contracts No. 11435003, No. 11225523, and No. 11521064. The work of Y.S. is supported by the Japan Society for the Promotion of Science (JSPS) Research Fellowships for Young Scientists, No. 16J08299. 


\section{APPENDIX: LOOP INTEGRAL FOR THE DIAGRAM IN FIG. 1(b)}

The expression of the up-quark CEDM $d_{u}^{C}$ in the mass insertion approximation is

$$
\begin{aligned}
d_{u}^{C} & =\frac{\alpha_{s}}{4 \pi} \frac{M_{\tilde{g}}}{m_{\tilde{t}}^{2}} \operatorname{Im}\left[\left(\delta_{L L}^{u}\right)_{31}\left(\delta_{R L}^{u}\right)_{33}\left(\delta_{R R}^{u}\right)_{13}\right] \times F_{\mathrm{MIA}}\left(r_{\tilde{g}}, r_{\tilde{t}}\right) \\
F_{\mathrm{MIA}}\left(r_{\tilde{g}}, r_{\tilde{t}}\right) & \equiv 6 r_{\tilde{t}}^{2}\left(-3 I_{G}\left(r_{\tilde{g}}, r_{\tilde{t}}\right)+\frac{1}{3} I_{S_{1}}\left(r_{\tilde{g}}, r_{\tilde{t}}\right)+\frac{1}{3} I_{S_{2}}\left(r_{\tilde{g}}, r_{\tilde{t}}\right)+\frac{1}{3} I_{S_{3}}\left(r_{\tilde{g}}, r_{\tilde{t}}\right)+\frac{1}{3} I_{S_{4}}\left(r_{\tilde{g}}, r_{\tilde{t}}\right)\right)
\end{aligned}
$$

where $r_{\tilde{g}}=\frac{M_{\tilde{g}}^{2}}{m_{\tilde{u}}^{2}}, r_{\tilde{t}}=\frac{m_{\tilde{t}}^{2}}{m_{\tilde{u}}^{2}}$ and $I_{i}\left(r_{\tilde{g}}, r_{\tilde{t}}\right)$ are loop integrals. Each integral is

$$
\begin{aligned}
I_{G}\left(r_{\tilde{g}}, r_{\tilde{t}}\right) & =\int_{0}^{1} d x_{1} \cdots d x_{4} \delta\left(\sum_{i} x_{i}-1\right) \frac{2 x_{1} x_{3} x_{4}}{\left[r_{\tilde{g}}\left(x_{1}+x_{2}\right)+x_{3}+r_{\tilde{t}} x_{4}\right]^{4}}, \\
I_{S_{1}}\left(r_{\tilde{g}}, r_{\tilde{t}}\right) & =\int_{0}^{1} d x_{1} \cdots d x_{4} \delta\left(\sum_{i} x_{i}-1\right) \frac{\left(2 x_{3}+2 x_{4}-1\right) x_{3} x_{4}}{\left[r_{\tilde{g}} x_{1}+x_{2}+x_{3}+r_{\tilde{t}} x_{4}\right]^{4}}, \\
I_{S_{2}}\left(r_{\tilde{g}}, r_{\tilde{t}}\right) & =\int_{0}^{1} d x_{1} \cdots d x_{5} \delta\left(\sum_{i} x_{i}-1\right) \frac{\left(2 x_{3}+2 x_{5}-1\right) x_{5}}{\left[r_{\tilde{g}} x_{1}+x_{2}+x_{3}+r_{\tilde{t}}\left(x_{4}+x_{5}\right)\right]^{4}}, \\
I_{S_{3}}\left(r_{\tilde{g}}, r_{\tilde{t}}\right) & =\int_{0}^{1} d x_{1} \cdots d x_{5} \delta\left(\sum_{i} x_{i}-1\right) \frac{\left(2 x_{3}+2 x_{5}-1\right) x_{4}}{\left[r_{\tilde{g}} x_{1}+x_{2}+x_{3}+r_{\tilde{t}}\left(x_{4}+x_{5}\right)\right]^{4}}, \\
I_{S_{4}}\left(r_{\tilde{g}}, r_{\tilde{t}}\right) & =\int_{0}^{1} d x_{1} \cdots d x_{4} \delta\left(\sum_{i} x_{i}-1\right) \frac{\left(2 x_{3}-1\right) x_{2} x_{4}}{\left[r_{\tilde{g}} x_{1}+x_{2}+x_{3}+r_{\tilde{t}} x_{4}\right]^{4}} .
\end{aligned}
$$

We show the values of $F_{\mathrm{MIA}}\left(r_{\tilde{g}}, r_{\tilde{t}}\right)$ with several values of mass ratio, $r_{\tilde{g}}$ and $r_{\tilde{t}}$ (see Table V). 


\begin{tabular}{|c||c|c|c|c|c|c|}
\hline$r_{\tilde{t}} \backslash r_{\tilde{g}}$ & $0.2^{2}$ & $0.3^{2}$ & $0.5^{2}$ & $1^{2}$ & $2^{2}$ & $5^{2}$ \\
\hline \hline $0.1^{2}$ & $4.1 \times 10^{-2}$ & $1.1 \times 10^{-2}$ & $1.8 \times 10^{-3}$ & $1.2 \times 10^{-4}$ & $6.9 \times 10^{-6}$ & $1.6 \times 10^{-7}$ \\
\hline $0.2^{2}$ & $2.4 \times 10^{-1}$ & $7.9 \times 10^{-2}$ & $1.6 \times 10^{-2}$ & $1.2 \times 10^{-3}$ & $6.9 \times 10^{-5}$ & $1.5 \times 10^{-6}$ \\
\hline $0.3^{2}$ & $5.3 \times 10^{-1}$ & $2.0 \times 10^{-1}$ & $4.6 \times 10^{-2}$ & $4.0 \times 10^{-3}$ & $2.4 \times 10^{-4}$ & $5.1 \times 10^{-6}$ \\
\hline $0.4^{2}$ & $8.5 \times 10^{-1}$ & $3.6 \times 10^{-1}$ & $9.1 \times 10^{-2}$ & $8.8 \times 10^{-3}$ & $5.6 \times 10^{-4}$ & $1.2 \times 10^{-5}$ \\
\hline $0.5^{2}$ & 1.2 & $5.2 \times 10^{-1}$ & $1.4 \times 10^{-1}$ & $1.5 \times 10^{-2}$ & $1.0 \times 10^{-3}$ & $2.1 \times 10^{-5}$ \\
\hline $0.6^{2}$ & 1.4 & $6.8 \times 10^{-1}$ & $2.0 \times 10^{-1}$ & $2.3 \times 10^{-2}$ & $1.6 \times 10^{-3}$ & $3.4 \times 10^{-5}$ \\
\hline $0.7^{2}$ & 1.7 & $8.2 \times 10^{-1}$ & $2.6 \times 10^{-1}$ & $3.2 \times 10^{-2}$ & $2.3 \times 10^{-3}$ & $5.0 \times 10^{-5}$ \\
\hline $0.8^{2}$ & 1.9 & $9.6 \times 10^{-1}$ & $3.2 \times 10^{-1}$ & $4.1 \times 10^{-2}$ & $3.2 \times 10^{-3}$ & $6.8 \times 10^{-5}$ \\
\hline $0.9^{2}$ & 2.1 & 1.1 & $3.7 \times 10^{-1}$ & $5.1 \times 10^{-2}$ & $4.1 \times 10^{-3}$ & $8.9 \times 10^{-5}$ \\
\hline $1^{2}$ & 2.3 & 1.2 & $4.2 \times 10^{-1}$ & $6.1 \times 10^{-2}$ & $5.0 \times 10^{-3}$ & $1.1 \times 10^{-4}$ \\
\hline $2^{2}$ & 3.2 & 1.8 & $7.6 \times 10^{-1}$ & $1.4 \times 10^{-1}$ & $1.5 \times 10^{-2}$ & $4.0 \times 10^{-4}$ \\
\hline $5^{2}$ & 3.7 & 2.3 & 1.0 & $2.4 \times 10^{-1}$ & $3.3 \times 10^{-2}$ & $1.2 \times 10^{-3}$ \\
\hline
\end{tabular}

Table V: The values of $F_{\mathrm{MIA}}\left(r_{\tilde{g}}, r_{\tilde{t}}\right)$ with several values of $r_{\tilde{g}}$ and $r_{\tilde{t}}$. 

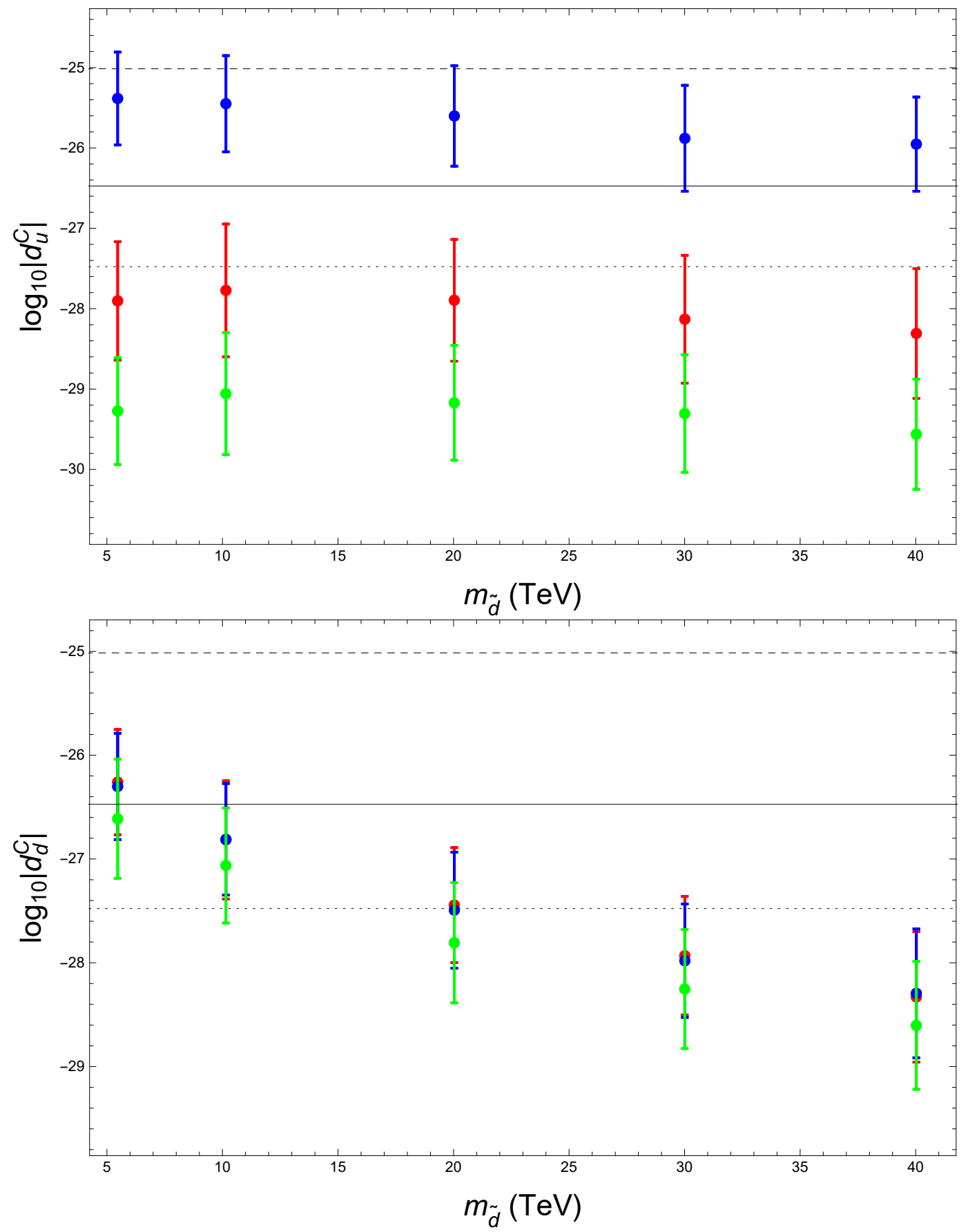

Figure 2: $m_{\tilde{d}}$ dependence of $d_{u}^{C}$ (upper panel) and $d_{d}^{C}$ (lower panel). Red, Blue and Green plots are real $Y_{u}$ type, complex $Y_{u}$ type and $E_{6}$ model, respectively. Each error bar shows the standard deviation for the predicted values of $\log _{{ }_{10}}\left|d_{q}^{C}\right|(q=u, d)$ which are obtained in various model points with different $O(1)$ coefficients. The black solid line is the current bound from $\mathrm{Hg}$ EDM and the allowed region is the lower area. The dashed line shows the current bound from the neutron EDM, and the dotted line is the bound expected in future experiments of neutron EDM. We choose $m_{3}$ and $M_{1 / 2}$ to become light stop at the SUSY scale $(1 \mathrm{TeV})$, and in these figures, we set $m_{\tilde{t}}=(2000 \pm 250) \mathrm{GeV}$. We also set $A_{0}=-1 \mathrm{TeV}$. 

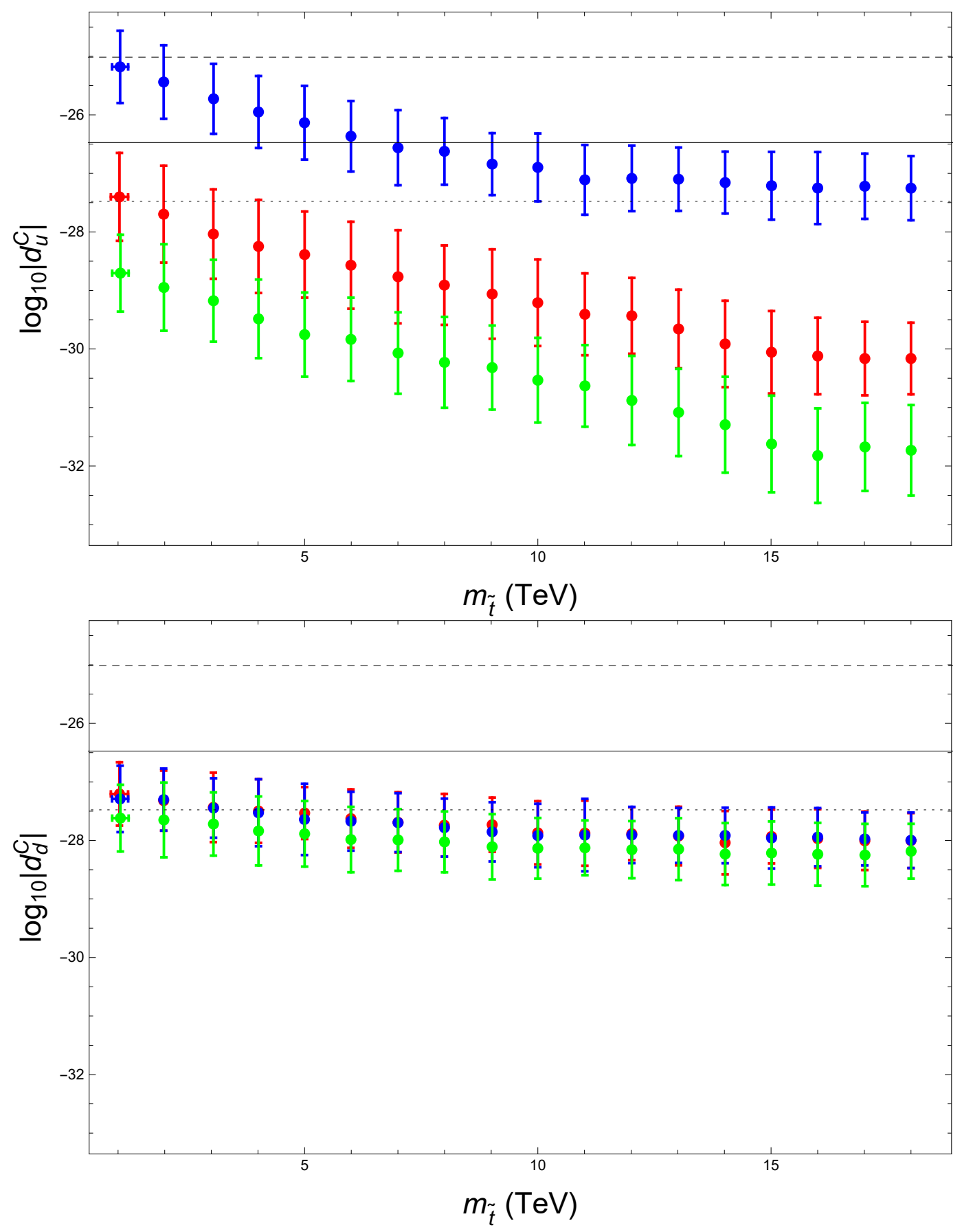

Figure 3: $m_{\tilde{t}}$ dependence of $d_{u}^{C}$ (upper panel) and $d_{d}^{C}$ (lower panel). The red, blue and green plots are real $Y_{u}$ type, complex $Y_{u}$ type and the $E_{6}$ model, respectively. Each vertical error bar shows the standard deviation for the predicted values of $\log _{10}\left|d_{q}^{C}\right|(q=u, d)$ which are obtained in various model points with different $O(1)$ coefficients. The horizontal error bar shows the distribution of stop masses by variation of $O(1)$ coefficients of Yukawa couplings and $A$ parameters. The black solid line is current bound from Hg EDM and allowed region is lower area. The dashed line shows the current bound from neutron EDM, and the dotted line is the bound expected in future experiments of neutron EDM. In these figures, we set $m_{0}=10 \mathrm{TeV}$ and $A_{0}=-1 \mathrm{TeV}$. 

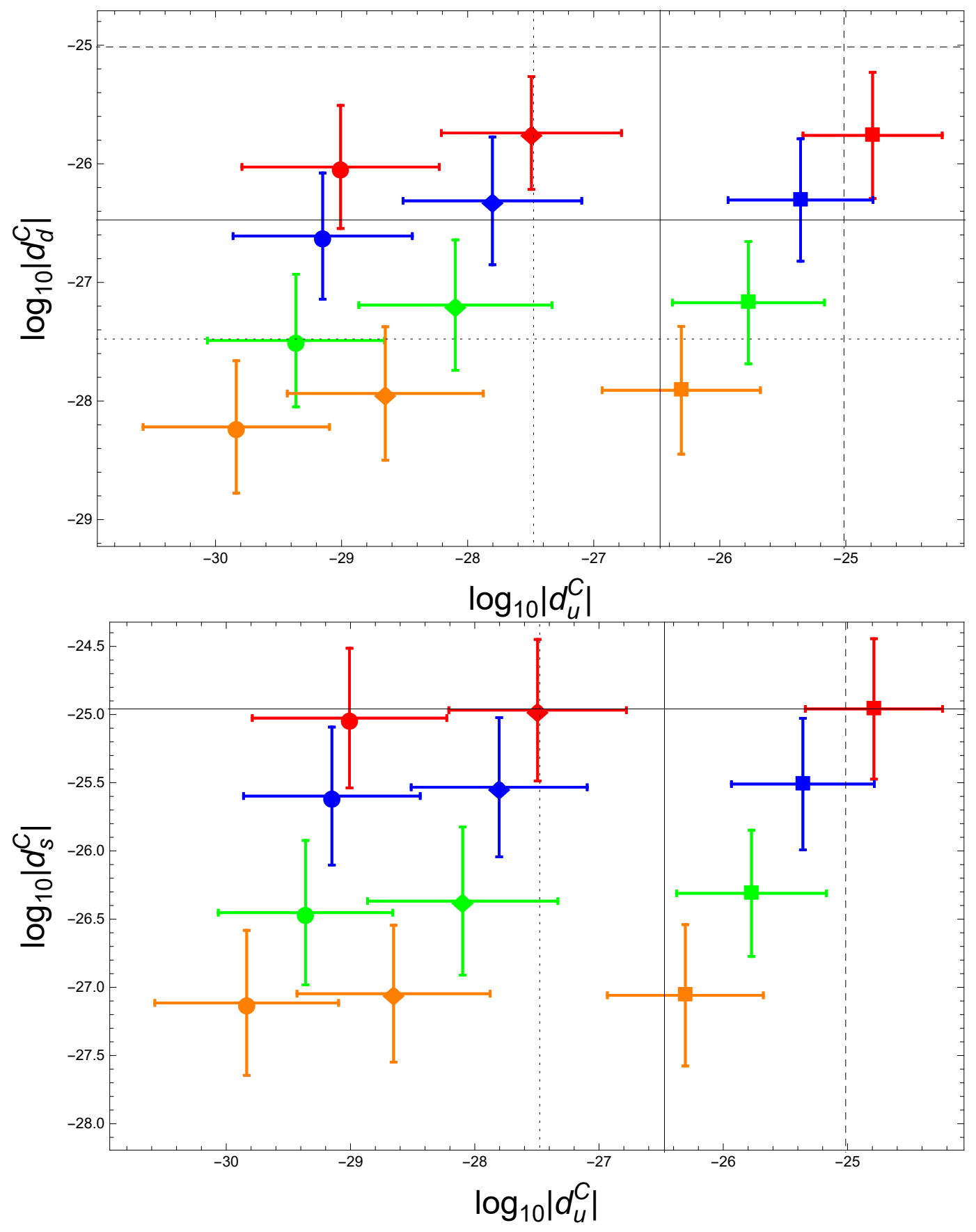

Figure 4: Up, down and strange quark CEDM in three type of boundary condition of $Y_{u}$. Upper panel is up and down quark CEDM and lower panel is up and strange quark CEDM. Diamond, square and circle plots are real $Y_{u}$ type, complex $Y_{u}$ type and $E_{6}$ model, respectively. Red, blue, green and orange mean that $m_{0}$ is $5 \mathrm{TeV}, 10 \mathrm{TeV}, 20 \mathrm{TeV}$ and $40 \mathrm{TeV}$. Each error bar shows the standard deviation for the predicted values of $\log _{10}\left|d_{q}^{C}\right|(q=u, d, s)$ which are obtained in various model points with different $O(1)$ coefficients. Black solid line is the current bound from $\mathrm{Hg}$ EDM and allowed region is lower left area. Dashed line shows the current bound from neutron EDM, and the dotted line is the bound expected in future experiments of neutron EDM. 


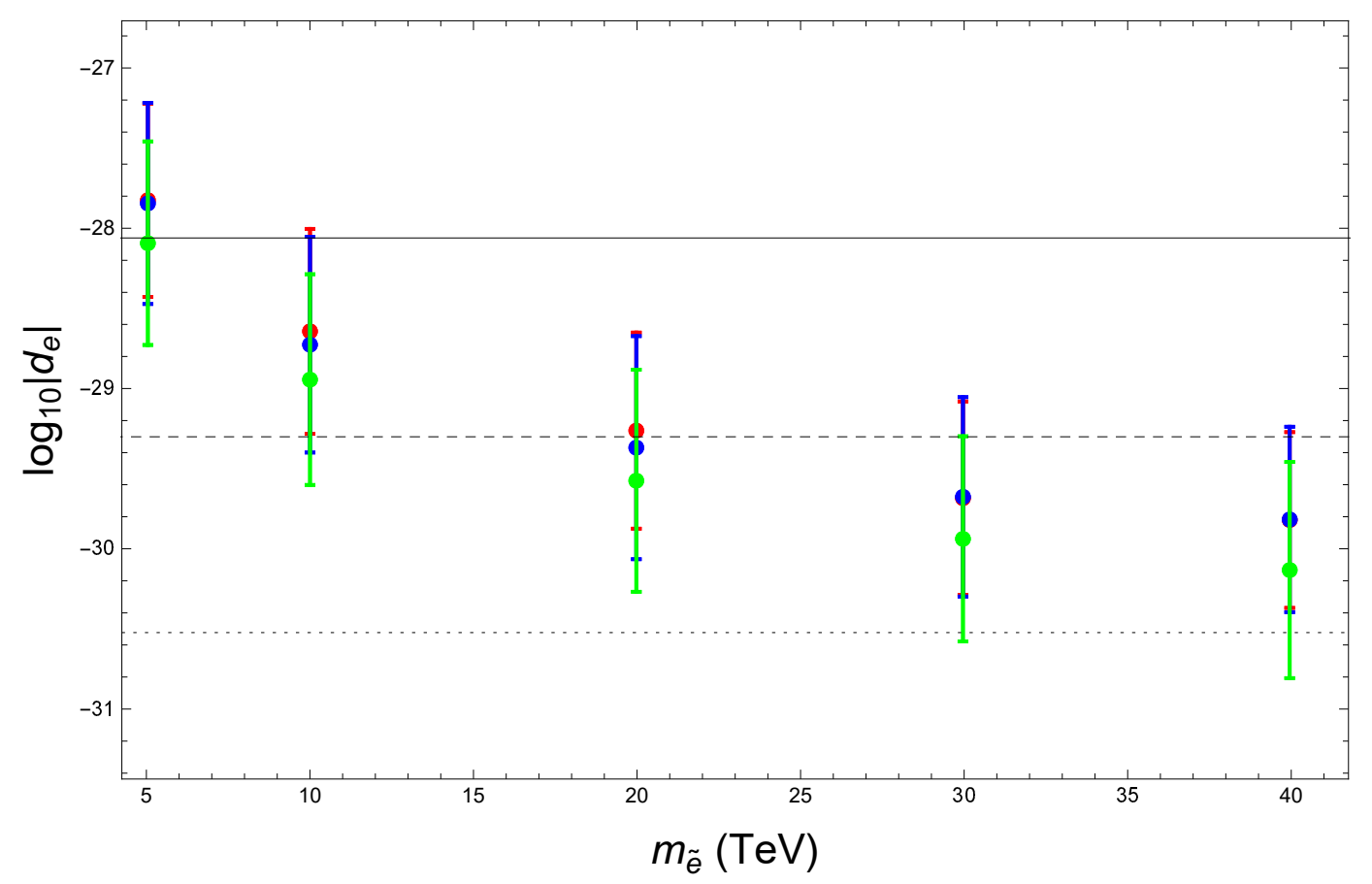

Figure 5: $m_{\tilde{e}}$ dependence of $d_{e}$ in three type of boundary condition of $Y_{u}$. Red, Blue and Green plots are real $Y_{u}$ type, complex $Y_{u}$ type and $E_{6}$ model, respectively. Each error bar shows the standard deviation for the predicted values of $\log _{10}\left|d_{e}\right|$ which are obtained in various model points with different $O(1)$ coefficients. Black solid line is current bound and allowed region is the lower area. Other input parameters are same as for the Fig. 2 . The dashed(dotted) line shows the future bound expected by ACME II (III)[28]. 


\section{References}

[1] H. Georgi and S. L. Glashow, Phys. Rev. Lett. 32, 438 (1974).

[2] F. Gabbiani, E. Gabrielli, A. Masiero, and L. Silvestrini, Nucl. Phys. B 477, 321 (1996) [hep-ph/9604387].

[3] G. Aad et al. [ATLAS Collaboration], Phys. Lett. B 716, 1 (2012) [arXiv:1207.7214 [hep-ex]]; S. Chatrchyan et al. [CMS Collaboration], Phys. Lett. B 716, 30 (2012) [arXiv:1207.7235 [hep-ex]].

[4] N. Arkani-Hamed and S. Dimopoulos, JHEP 0506, 073 (2005) [hep-th/0405159]; G. F. Giudice and A. Romanino, Nucl. Phys. B 699, 65 (2004) [Erratum-ibid. B 706, 487 (2005)] [hep-ph/0406088]; N. Arkani-Hamed, S. Dimopoulos, G. F. Giudice, and A. Romanino, Nucl. Phys. B 709, 3 (2005) [hep-ph/0409232]; J. D. Wells, Phys. Rev. D 71, 015013 (2005) [hep-ph/0411041]; G. F. Giudice and A. Strumia, Nucl. Phys. B 858, 63 (2012) [arXiv:1108.6077 [hep-ph]]; L. J. Hall and Y. Nomura, JHEP 1201, 082 (2012) [arXiv:1111.4519 [hep-ph]]; M. Ibe and T. T. Yanagida, Phys. Lett. B 709, 374 (2012) [arXiv:1112.2462 [hep-ph]]; M. Ibe, S. Matsumoto, and T. T. Yanagida, Phys. Rev. D 85, 095011 (2012) [arXiv:1202.2253 [hep-ph]]; N. Arkani-Hamed, A. Gupta, D. E. Kaplan, N. Weiner, and T. Zorawski, arXiv:1212.6971 [hep-ph].

[5] S. Dimopoulos and G. Giudice, Phys. Lett. B 357, 573 (1995) [hep-ph/9507282]; A. Pomarol and D. Tommasini, Nucl. Phys. B 466, 3 (1996) [hep-ph/9507462]; A. Cohen, D. Kaplan, and A. Nelson, Phys. Lett. B 388, 588 (1996) [hep-ph/9607394]; N. Arkani-Hamed and H. Murayama, Phys. Rev. D 56, 6733 (1997) [hep-ph/9703259]; J. Hisano, K. Kurosawa, and Y. Nomura, Nucl. Phys. B584, 3 (2000) [hep$\mathrm{ph} / 0002286]$.

[6] N. Maekawa, Phys. Lett. B 561, 273 (2003) [hep-ph/0212141]; Prog. Theor. Phys. 112, 639 (2004) [hep-ph/0402224].

[7] K. Hamaguchi, M. Kakizaki, and M. Yamaguchi, Phys. Rev. D 68, 056007 (2003) [hep-ph/0212172].

[8] H. Fritzsch and P. Minkowski, Annals Phys. 93, 193 (1975); F. Gursey, P. Ramond, and P. Sikivie, Phys. Lett. B 60, 177 (1976); Y. Achiman and B. Stech, Phys. Lett. B 77, 389 (1978); R. Barbieri and D. V. Nanopoulos, Phys. Lett. B 91, 369 (1980).

[9] T. Kugo and J. Sato, Prog. Theor. Phys. 91, 1217 (1994) [hep-ph/9402357]; N. Irges, S. Lavignac, and P. Ramond, Phys. Rev. D 58, 035003 (1998) [hep-ph/9802334]; M. Bando and T. Kugo, Prog. Theor. Phys. 101, 1313 (1999) [hep-ph/9902204]; M. Bando, T. Kugo, and K. Yoshioka, Prog. Theor. Phys. 104, 211 (2000) [hepph/0003220]; 
[10] M. Bando and N. Maekawa, Prog. Theor. Phys. 106, 1255 (2001) [hep-ph/0109018]; N. Maekawa and T. Yamashita, Prog. Theor. Phys. 107, 1201 (2002) [hep$\mathrm{ph} / 0202050]$.

[11] N. Maekawa, Prog. Theor. Phys. 106, 401 (2001) [hep-ph/0104200]; Prog. Theor. Phys. 107, 597 (2002) [hep-ph/0111205].

[12] S. -G. Kim, N. Maekawa, A. Matsuzaki, K. Sakurai, and T. Yoshikawa, Phys. Rev. D 75, 115008 (2007) [hep-ph/0612370]; Prog. Theor. Phys. 121, 49 (2009) [arXiv:0803.4250 [hep-ph]].

[13] J. M. Pendlebury et al., Phys. Rev. D 92, no. 9, 092003 (2015) [arXiv:1509.04411 [hep-ex]]; B. Graner, Y. Chen, E. G. Lindahl, and B. R. Heckel, Phys. Rev. Lett. 116, 161601 (2016) [arXiv:1601.04339].

[14] S. Dimopoulos and L. J. Hall, Phys. Lett. B 344, 185 (1995) [hep-ph/9411273]; J. Hisano and Y. Shimizu, Phys. Rev. D 70, 093001 (2004) [hep-ph/0406091].

[15] M. Ishiduki, S. -G. Kim, N. Maekawa, and K. Sakurai, Prog. Theor. Phys. 122, 659 (2009) [arXiv:0901.3400 [hep-ph]]; F. Sala, JHEP 1403, 061 (2014) [arXiv:1312.2589 [hep-ph]].

[16] L. J. Hall, V. A. Kostelecky, and S. Raby, Nucl. Phys. B 267, 415 (1986).

[17] K. Fuyuto, J. Hisano, and N. Nagata, Phys. Rev. D 87, no. 5, 054018 (2013) [arXiv:1211.5228 [hep-ph]].

[18] M. Jung and A. Pich, JHEP 1404, 076 (2014) [arXiv:1308.6283 [hep-ph]].

[19] J. Engel, M. J. Ramsey-Musolf, and U. van Kolck, Prog. Part. Nucl. Phys. 71, 21 (2013) [arXiv:1303.2371 [nucl-th]].

[20] M. Ishiduki, S. -G. Kim, N. Maekawa, and K. Sakurai, Phys. Rev. D 80, 115011 (2009) [Erratum-ibid. D 81, 039901 (2010)] [arXiv:0910.1336 [hep-ph]]; H. Kawase and N. Maekawa, Prog. Theor. Phys. 123, 941 (2010) [arXiv:1005.1049 [hep-ph]]; N. Maekawa and K. Takayama, Phys. Rev. D 85, 095015 (2012) [arXiv:1202.5816 [hep-ph]].

[21] Y. Nakai and M. Reece, arXiv:1612.08090 [hep-ph].

[22] S. P. Martin and M. T. Vaughn, Phys. Rev. D 50, 2282 (1994); Phys. Rev. D 78, 039903(E) (2008)[hep-ph/9311340].

[23] C. D. Froggatt and H. B. Nielsen, Nucl. Phys. B 147, 277 (1979); L. E. Ibanez and G. G. Ross, Phys. Lett. B 332, 100 (1994) [hep-ph/9403338]. 
[24] K. Bodek et al., arXiv:0806.4837 [nucl-ex]; I. Altarev et al., Nucl. Instrum. Meth. A 611, 133 (2009); C. A. Baker et al., J. Phys. Conf. Ser. 251, 012055 (2010); D. H. Beck et al. [nEDM Collaboration], arXiv:1111.1273 [nucl-ex]; I. Altarev et al., Nuovo Cim. C035N04, 122 (2012).

[25] S. Heinemeyer, W. Hollik, and G. Weiglein, Eur. Phys. J. C 9 (1999) 343 [hepph/9812472]; Comput. Phys. Commun. 124 (2000) 76 [hep-ph/9812320]; G. Degrassi, S. Heinemeyer, W. Hollik, P. Slavich, and G. Weiglein, Eur. Phys. J. C 28 (2003) 133 [hep-ph/0212020]; M. Frank, T. Hahn, S. Heinemeyer, W. Hollik, H. Rzehak, and G. Weiglein, JHEP 0702 (2007) 047 [hep-ph/0611326]; T. Hahn, S. Heinemeyer, W. Hollik, H. Rzehak, and G. Weiglein, Comput. Phys. Commun. 180 (2009) 1426. See http://www.feynhiggs.de.

[26] J. Baron et al. (ACME Collaboration), Science 343, 269 (2014).

[27] J. Ellis, J. S. Lee, and A. Pilaftsis, JHEP 0810 (2008) 049, [arXiv:0808.1819 [hep-ph]].

[28] T. Chupp and M. Ramsey-Musolf, Phys. Rev. C 91, 035502 (2015); J. Doyle, http://online.kitp.ucsb.edu/online/nuclear_c16/doyle/. Talk at the KITP Conference: Symmetry Tests in Nuclei and Atomes. 